\title{
Aspectos reproductivos, arquitectura y fenomorfología de Maytenus boaria Molina (Celastraceae) en Chile central
}

\author{
Reproductives aspects, architecture and phenomorphology of Maytenus boaria Molina \\ (Celastraceae) in central Chile
}

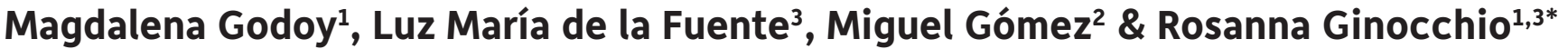

\author{
Departamento de Ecosistemas y Medio Ambiente, Facultad de Agronomía e Ingeniería Forestal, Pontificia Universidad Católica de Chile, Santiago, Chile. \\ ${ }^{2}$ Departamento de Ciencias Vegetales, Facultad de Agronomía e Ingeniería Forestal, Pontificia Universidad Católica de Chile, Santiago, Chile. \\ ${ }^{3}$ Center of Applied Ecology and Sustainability (CAPES), Facultad de Ciencias Biológicas, Pontificia Universidad Católica de Chile, Santiago, Chile. \\ *rginocch@uc.cl
}

\section{RESUMEN}

Maytenus boaria Molina (Celastraceae) es un árbol nativo siempreverde de amplia distribución en Chile, con alto potencial económico por los diversos usos tradicionales descritos. A pesar de esto, existe escasa y confusa información biológica de la especie. El objetivo de este estudio fue ampliar el conocimiento sobre aspectos reproductivos y el sistema sexual de M. boaria, además de la arquitectura de la copa y su fenomorfología. El estudio se realizó entre agosto y diciembre de 2016, con individuos adultos ( $n=17)$ seleccionados en sectores urbanos de la Región Metropolitana. Se realizó un ensayo de polinización y se identificó el sexo de las flores de cada árbol seleccionado. Además, se realizó un diagrama fenomorfológico de la especie a través de la interpretación modular de la arquitectura y la identificación de sus fenofases. Los resultados indican que $M$. boaria es dioica y andromonoica; presenta una baja autogamia autónoma y es una especie no apomíctica que necesita de vectores de polinización para la producción de semillas. Posee cuatro módulos arquitectónicos en su copa -dolicoblasto de yema apical, dolicoblasto de yema axilar, inflorescencia y braquiblasto temporal reproductivo- dispuestos en dos unidades arquitecturales. Los aspectos reproductivos analizados y la fenomorfología de esta especie indican que estaría en un proceso evolutivo respecto a la funcionalidad de las flores hermafroditas, lo que ha sido planteado como un paso intermedio del hermafroditismo a la dioecia. Un mayor conocimiento de estas temáticas permitirá un mejor entendimiento de la autoecología de la especie, para un adecuado manejo productivo de esta.

Palabras clave: autogamia autónoma, ciclo fenológico, dioecia, fenofases, maitén.

\section{ABSTRACT}

Maytenus boaria Molina (Celastraceae) is an evergreen native tree which is widely distributed in Chile. It has a high economic potential due to its medicinal value, among others. Despite this, biological information about the species is scarce and often confusing. The objective of the study was to broaden the knowledge about reproductive aspects and the sexual system of M. boaria, along with its modular architecture and phenomorphology. The study was conducted from August through December of 2016, with adults plants $(n=17)$ selected in urban areas of the Región Metropolitana. A pollination test was performed and the sex of the flowers of each selected tree was identified. In addition, a pheno-morphological diagram of the species was made through the modular interpretation of its architecture and the identification of its phenophases. Results showed that M. boaria is a dioecious and andromonoecious species; it has a low autonomous autogamy and is non-apomictic species that needs pollination vectors for seed production. It has four architectural modules in 
its crown: dolichoblast of apical bud, dolichoblast of axillary bud, inflorescence and reproductive temporary brachyblasts which are distributed in two architectonic units. The floral biology and pheno-morphology of this species indicate that it would be in an evolutionary process regarding the functionality of hermaphroditic flowers, which has been proposed as an intermediate step from hermaphroditism to dioecy. Further knowledge of these topics will allow for a better understanding of the autoecology of the species in order to have an appropriate and productive management of it.

Keywords: autonomous autogamy, dioecious, Mayten tree, phenology cycle, phenophases.

\section{INTRODUCCIÓN}

Maytenus boaria Molina, el maitén, es una especie arbórea siempreverde de la familia Celastraceae, nativo de Chile, Argentina, Perú y Brasil (Rodríguez et al. 1983, Montenegro et al. 1989, Pirani \& De Carvalho-Okano 1999, Rodríguez et al. 2018). Dentro del territorio nacional posee una amplia distribución geográfica, la que se extiende desde la Provincia de Huasco (Región de Atacama) a la Provincia del Capitán Prat (Región de Aysén) (Rodríguez et al. 1983). A su vez, posee una amplia distribución altitudinal, ya que crece en ambas cordilleras y en el valle central, demostrándose así su gran plasticidad (Di Castri \& Hajek 1976, Rodríguez et al. 1983). En Chile, además de M. boaria, se encuentran otras tres especies del género: Maytenus disticha Urb., Maytenus magellanica Hook. f. y Maytenus chubutensis (Speg.) Lourteig, O’Donell \& Sleumer (Rodríguez et al. 2018). Estas especies pertenecen a un clado del género Maytenus que agrupa a 15-24 especies de linaje andino-subtropical las que comparten caracteres morfológicos comunes. Entre ellos, la presencia de inflorescencias axilares fasciculadas o cimosas a veces reducidas a flores unisexuales, frutos capsulares con pericarpio membranáceo con 2-3 valvas y semillas con arilos rojos o amarillentos que cubren total o parcialmente las semillas (Biral et al. 2017). Este género es el más abundante e importante de la familia Celastraceae (Santos et al. 2007), con alrededor de 140 especies cuyo centro de diversidad se encuentra en Sudamérica, siendo el maitén la especie tipo (Loesener 1942, Biral et al. 2017).

El maitén, al ser una especie secundaria en los diversos bosques en que participa (Luebert \& Pliscoff 2017), no ha despertado mayor interés, a pesar de que podría ser adecuada, por ejemplo, como especie ornamental urbana (Riedemann \& Aldunate 2001, Dobbs et al. 2011, Santilli et al. 2018), para un sistema de silvopastoreo ya que tanto sus hojas palatables como la madera pueden ser cosechadas (Donoso \& Wendler 1985, Donoso et al. 2006). También hay que destacar el alto valor medicinal de sus hojas (Riedemann \& Aldunate 2001, Hoffmann 2005, Zapata et al. 2006) y la gran cantidad de ácidos grasos que se pueden extraer de sus semillas (Gusinde 1917, Hoffmann \& Kummerow 1962, Tinto 1977, Menegoz \& Zapata 2017). A pesar de sus características y usos potenciales productivos, no se le ha dado mayor importancia, existiendo escasa y/o confusa información sobre aspectos de su biología básica, los que podrían potenciar su uso, como lo son su sistema sexual, aspectos reproductivos, arquitectura y fenomorfología. Por ejemplo, el sistema sexual de M. boaria es aún una incógnita o no ha sido debidamente interpretada, ya que no existe consenso entre los autores que la han estudiado. Algunos la han descrito como una especie monoica o poligamodioica (Muñoz 1966, Rodríguez et al. 1983, Montenegro et al. 1989, Riedemann \& Aldunate 2001, Barrera \& Meza 2006), mientras que otros la definen como poligamomonoica (Hoffmann et al. 2003, Hoffmann 2005, Donoso et al. 2006) o bien como dioica (Donoso \& Wendler 1985, Arroyo \& Uslar 1993, Dawson 2016).

Conocer e interpretar la sexualidad floral permitirá tener un mejor entendimiento de la especie, ya que esta incide sobre la transferencia de polen, el sistema reproductivo y el tipo de polinizadores que estarían asociados a la especie (Primack 1985, Zimmerman 1988, Lloyd \& Schoen 1992, Barrett 2003). Todos estos aspectos son relevantes de conocer para una adecuada domesticación y el posterior cultivo productivo de esta.

El potencial manejo productivo de la especie también depende del crecimiento característico de la copa, principalmente en cuanto al incremento de la biomasa y el arreglo arquitectural de esa biomasa en el espacio (Küppers 1989). El estudio de la arquitectura modular entrega información básica de gran importancia en el ámbito ecológico (Meusel et al. 1977) y de la silvicultura del bosque nativo. Por ello, la selección de ciertas características arquitecturales puede conducir a un mayor rendimiento productivo si se lleva a la especie a cultivo o domesticación, especialmente en el rendimiento de semillas y frutos (Wang et al. 2018). Por otra parte, la fenología de la copa, que corresponde al estudio de la secuencia temporal de eventos biológicos recurrentes en 
especies vegetales, se ha relacionado con la morfología de estas, siendo clave para interpretar los cambios temporales en el crecimiento vegetativo y reproductivo de la especie en un ambiente determinado (Orshan 1989) y, por ende, de los manejos que la especie requerirá en un sistema de cultivo.

El objetivo de este estudio fue ampliar el conocimiento sobre aspectos reproductivos y el sistema sexual de M. boaria, considerando la escasa y confusa información existente en la literatura. Además, se buscó analizar la arquitectura de la especie desde el enfoque de la arquitectura modular y su fenomorfología. Un mayor conocimiento de estas temáticas permitirá un mejor entendimiento de la autoecología de la especie y, por ende, un adecuado manejo productivo de la misma, considerando los potenciales usos que esta posee. Esta investigación aporta información que facilita y sirve de punto de partida para futuros estudios y nuevas líneas de investigación sobre M. boaria.

\section{MATERIALES Y MÉTODOS}

\section{ÁREA DE ESTUDIO}

El estudio se llevó a cabo entre los meses de agosto y diciembre de 2016, con individuos adultos seleccionados en sectores urbanos (p. ej., calles y plazas) de las comunas de Ñuñoa y Macul, ciudad de Santiago, Región Metropolitana, Chile $\left(32^{\circ} 55^{\prime}-34^{\circ} 19^{\prime} \mathrm{S}\right.$ y $\left.69^{\circ} 47^{\prime}-71^{\circ} 43^{\prime} \mathrm{O}\right)$. El área se encuentra bajo la influencia de un macrobioclima mediterráneo, el cual se caracteriza por tener un verano muy seco y un invierno muy lluvioso, con una temperatura media anual de $14,2^{\circ} \mathrm{C}$ y un promedio anual de precipitaciones de $335 \mathrm{~mm}$ (Luebert \& Pliscoff 2017).

\section{ELECCIÓN Y CARACTERIZACIÓN DE INDIVIDUOS}

Dentro del área de estudio se seleccionaron 17 individuos adultos al azar. Posteriormente fueron clasificados como predominantes femeninos $(n=7)$ o predominantes masculinos $(n=10)$, según la dominancia del sexo de las flores presentes en el árbol. Todos los individuos se encontraban en el mismo estado ontológico (madurez) y fenológico, además de presentar un buen estado fitosanitario. A cada individuo se le midió la altura con un hipsómetro, el diámetro a la altura del pecho (DAP) con una forcípula a 1,3 $\mathrm{m}$ del nivel del suelo y el diámetro de copa de norte a sur (N-S) y de este a oeste (E-O) con una huincha métrica.

\section{AsPECTOS REPRODUCTIVOS}

Para evaluar el sistema reproductivo de una especie se requiere realizar cinco tratamientos de polinización: agamospermia, autopolinización espontánea (no asistida), autopolinización, polinización cruzada y natural/control (Eckert et al. 2010, Cuartas-Domínguez et al. 2017, RiveraHutinel \& Acevedo-Orellana 2017). Con el propósito de analizar parte del sistema reproductivo que posee M. boaria, se realizaron dos de ellos; autopolinización espontánea (no asistida) y polinización natural (control). El tratamiento de autopolinización espontánea evalúa la autogamia autónoma, es decir, la producción de semillas sin intervención de polinizadores (Larson \& Barrett 2000, Eckert et al. 2010, Cuartas-Domínguez et al. 2017). Para este caso, las ramillas seleccionadas, con yemas florales intactas, se aislaron completamente con bolsas de organza semipermeables de $20 \mathrm{~cm}$ de largo, hasta la cosecha de frutos (Herrera 1987). En cambio, en el control las flores quedaron expuestas a condiciones naturales de polinización. Para este ensayo se escogieron nueve árboles al azar, tres predominantes femeninos (flores pistiladas) y seis predominantes masculinos (flores estaminadas). De cada individuo se seleccionaron al azar dos ramillas de un año de edad y $20 \mathrm{~cm}$ de longitud, una para cada tratamiento. La edad de las ramillas se pudo determinar por la presencia de yemas florales, las cuales se forman en el periodo de crecimiento previo (Montenegro et al. 1989). En ambos tratamientos se registró el número de yemas florales al inicio del ensayo y el número de frutos formados al finalizar el ensayo, para así calcular el porcentaje de fructificación.

\section{DesCRIPCIÓN DEL SISTEMA SEXUAL}

Para evaluar el sistema sexual de la especie (sexualidad floral), se escogieron 10 árboles al azar, de los cuales tres correspondían a individuos predominantes femeninos y siete a individuos predominantes masculinos. El estudio se realizó en dos oportunidades dentro del periodo de floración de la especie, a fines de agosto de 2016 y a fines de septiembre del mismo año. De cada individuo se seleccionaron cinco ramas al azar y de cada una de ellas se analizaron cuatro ramillas de un año edad. En cada ramilla se analizaron las flores de un segmento reproductivo de 10 nudos, bajo una lupa estereoscópica Variscope con magnitud 40X. El sexo de las flores se identificó por medio de descripciones morfológicas descritas en la literatura (Rodríguez et al. 1983, Lourteig 1988), evaluándose también el porcentaje de antesis según el sexo. El género Maytenus posee flores hermafroditas (perfectas) o unisexuales (Navas 1976). En M. boaria se pueden encontrar flores femeninas o pistiladas que presentan un ovario desarrollado y funcional con estambres rudimentarios, por su parte las flores masculinas o estaminadas presentan ovario atrofiado y estambres desarrollados y funcionales (Navas 1976, Rodríguez et al. 1983). 


\section{Arquitectura}

Para el estudio de la arquitectura, se realizó una interpretación modular de esta, durante el período de crecimiento vegetativo y reproductivo de la especie. Para ello se identificaron y contabilizaron los distintos tipos de módulos que se desarrollaron a partir de las yemas apicales y axilares. A su vez, se analizó la distribución espacial de los diferentes módulos, determinándose y contabilizándose los distintos tipos de unidades arquitecturales que se desarrollan en la copa de M. boaria (Ginocchio \& Montenegro 1992).

El muestreo se realizó en terreno, entre septiembre y noviembre de 2016. Se seleccionaron ocho individuos en total, cuatro predominantes femeninos y cuatro predominantes masculinos. De cada individuo se analizaron cuatro ramas escogidas al azar $(n=32)$, de entre 3 y 4 años de edad, una para cada orientación de la copa, utilizando escalera de tijera para alcanzar las ramillas en altura de forma manual. La edad de las ramillas se determinó identificando el acortamiento de la distancia entre metámeros (zona de entrenudos cortos), lo cual corresponde a la fase de reposo del alargamiento de la ramilla, es decir, al cambio de temporada de crecimiento (Tourn et al. 1999). Además, se registró y contabilizó la muerte apical, la cual se consideró como parte de la interpretación modular de las yemas apicales (Ginocchio \& Montenegro 1992).

\section{FenOMORFOLOGÍA}

Para el análisis fenomorfológico se realizó un seguimiento semanal de las mismas ramas utilizadas en el estudio de la arquitectura, durante los meses de agosto y diciembre de 2016. Se llevó a cabo un registro fotográfico y escrito de las fases fenológicas según Orshan (1989), a través de la presencia o ausencia de: el crecimiento vegetativo de dolicoblasto, el crecimiento vegetativo de braquiblastos, la floración y la formación de frutos. El estudio de estas fenofases se complementó con el estudio fenomorfológico realizado por Montenegro et al. (1989), el cual incluye las fenofases que no se alcanzaron a analizar en este estudio; como la formación de yemas florales, la dispersión de semillas y el desprendimiento de hojas de dolicoblastos.

\section{ANÁLISIS ESTADÍSTICO}

AsPeCtOS REPRODUCTIVOS

Para comparar los resultados de porcentajes de fructificación entre los tratamientos de autopolinización y polinización natural, se realizó la prueba para la diferencia de proporciones en el programa estadístico Infostat (Di Rienzo et al. 2016). Esta prueba se realizó por separado para los individuos predominantes femeninos y predominantes masculinos.

\section{Arquitectura}

La frecuencia de conteo de los distintos tipos de módulos y unidades arquitecturales identificadas se comparó entre sexos predominantes, así como también dentro de cada sexo. La frecuencia de conteo de los módulos se analizó de forma independiente para cada tipo de yema, apicales y axilares. Para conocer la existencia de dependencia entre tipo de sexo predominante y el tipo de módulo o unidad arquitecturales, se utilizó la prueba de Chi-cuadrado de homogeneidad ( $\chi^{2}$ con $a=0,05$ ) (Dytham 2011). Considerando que la prueba de normalidad de Shapiro-Wilks $(a=0,10)$ arrojó un comportamiento no normal, se realizaron pruebas no paramétricas. Para la comparación de medianas entre individuos predominantes femeninos y predominantes masculinos, los datos fueron analizados mediante una prueba $U$ de Mann-Whitney $(a=0,05)$. Esta misma prueba fue utilizada dentro de cada sexo, para la comparación de frecuencia de conteo de módulos en yemas apicales (incluyendo muerte apical) y unidades arquitecturales. En el caso particular de módulos en yemas axilares, se aplicó una prueba de Kruskal-Wallis $(a=0,05)$, con la prueba de Nemenyi, como prueba a posteriori para comparaciones múltiples. Los análisis estadísticos fueron realizados en el software R versión 2.11.1 (R Development Core Team 2010).

\section{RESULTADOS}

\section{Caracterización de individuos de Maytenus boaria}

El promedio de la altura de los individuos seleccionados de M. boaria fue 6,6 m (Tabla 1) y su rango fluctuó entre 4,5 y 7,5 $\mathrm{m}$, a excepción de dos individuos predominantes femeninos (EE y PPE) (Tabla 1). El promedio del diámetro a la altura del pecho (DAP) de los individuos seleccionados fue $18,6 \mathrm{~cm}$ y el rango general varió entre 10,4 y $22,0 \mathrm{~cm}$, exceptuando un individuo predominante femenino que alcanzó los $54,0 \mathrm{~cm}$ (PPE). El promedio de diámetro de copa de N-S y E-O fue 4,6 y 4,7 $\mathrm{m}$, respectivamente (Tabla 1 ).

\section{AsPECTOS REPRODUCTIVOS}

El porcentaje promedio de fructificación en individuos predominantes femeninos difirió significativamente entre los tratamientos (Prueba para la diferencia de proporciones $p<0,05)$. Las flores del tratamiento de autopolinización espontánea arrojaron un nulo porcentaje promedio de fructificación; en cambio, las del tratamiento de polinización natural, obtuvieron $46 \%$ de fructificación (Tabla 2). En cuanto a los individuos predominantes masculinos, los tratamientos de autopolinización y polinización natural no presentaron diferencias significativas (Prueba para la diferencia de 
proporciones $p=0,09$ ). En ambos casos se obtuvieron bajos porcentajes promedio de fructificación, siendo de 1,2\% para el tratamiento de autopolinización espontánea y un $0,3 \%$ en el de polinización natural (Tabla 2), lo que sugiere la presencia de órganos femeninos en estos individuos.

\section{Sistema SEXUAL}

Según la presencia y desarrollo de los órganos sexuales se identificaron tres tipos de flores: masculinas (estaminadas) (Fig. 1a), femeninas (pistiladas) (Fig. 1b) y hermafroditas o perfectas (Fig. 1c y 1d). Los individuos caracterizados como predominantes femeninos solo presentaron flores femeninas. En el caso de individuos caracterizados como predominantes masculinos se dieron dos condiciones: individuos que solo presentaron flores masculinas e individuos que presentaron tanto flores masculinas como hermafroditas, condición conocida como andromonoica. En estos últimos, las flores hermafroditas solo se encontraron en algunas ramillas, distribuidas de forma irregular a lo largo de estas, coexistiendo con flores masculinas en un mismo fascículo.
Aunque en este estudio no se realizó un análisis detallado de la morfología floral de la especie, es importante mencionar que se observó un tipo de dimorfismo floral en las flores hermafroditas. Por un lado, se identificó un morfo floral en que las anteras se encuentran por debajo del estigma (Fig. 1c) y un segundo morfo floral con anteras posicionadas a la misma altura del estigma (Fig. 1d).

Los porcentajes promedios de antesis en individuos predominantes femeninos fueron de un $33 \%$ a finales de agosto y un $79 \%$ a finales de septiembre (Tabla 3). En el caso de los individuos predominantes masculinos se presentaron porcentajes promedio de antesis en flores masculinas, en ambos muestreos, con $57 \%$ a finales de agosto y $88 \%$ a finales de septiembre (Tabla 3). Sin embargo, la antesis de flores hermafroditas para estos mismos individuos se dio exclusivamente en el segundo muestreo, a finales de septiembre, con un porcentaje promedio de 6\% (Tabla 3). En este caso, se detectó un amplio rango de porcentaje de antesis $(1,25 \%$ - 14,97\%) quedando en evidencia la variación porcentual entre individuos predominantes masculinos en cuanto a la antesis de flores hermafroditas.

TABLA 1. Caracterización de los individuos seleccionados de Maytenus boaria según sexo predominante (femenino o masculino), altura $(\mathrm{m})$, DAP $(\mathrm{cm})$ y diámetro de copa $(\mathrm{m})$. / Characterization of selected individuals of Maytenus boaria according to predominant sex (male or female), height $(\mathrm{m}), \mathrm{DBH}(\mathrm{cm})$ and canopy diameter $(\mathrm{m}) .{ }^{*}$ Error estándar. / Standard error.

\begin{tabular}{cccccc}
\hline \multirow{2}{*}{ Individuo } & Sexo predominante & Altura $(\mathbf{m})$ & DAP $(\mathbf{c m})$ & \multicolumn{2}{c}{ Diámetro de copa $(\mathbf{m})$} \\
\hline FA & Femenino & 6,0 & 22,0 & 4,9 & 5,2 \\
TB & Femenino & 6,0 & 20,0 & 5,4 & 4,8 \\
EE & Femenino & 12,0 & 17,8 & 5,5 & 5,7 \\
PPE & Femenino & 10,5 & 54,0 & 4,8 & 5,7 \\
HS & Femenino & 6,5 & 18,0 & 4,9 & 5,6 \\
HN & Femenino & 6,0 & 18,0 & 5,1 & 4,7 \\
CS & Femenino & 6,5 & 22,0 & 3,3 & 3,6 \\
TS & Masculino & 6,5 & 21,8 & 4,4 & 5,8 \\
CE & Masculino & 7,5 & 15,0 & 5,6 & 5,2 \\
EI & Masculino & 5,0 & 16,9 & 4,6 & 4,4 \\
PPPCA & Masculino & 6,0 & 16,5 & 3,6 & 5,2 \\
PPPPA & Masculino & 4,5 & 10,8 & 3,1 & 4,1 \\
CB & Masculino & 6,0 & 10,8 & 5,4 & 3,4 \\
PC & Masculino & 7,0 & 18,8 & 4,4 & 3,3 \\
LN & Masculino & 5,0 & 11,7 & 4,5 & 4,9 \\
HA & Masculino & 6,0 & 12,2 & 5,5 & 4,8 \\
CP & Masculino & 5,5 & 10,4 & 3,3 & 3,6 \\
\hline & Promedio & 6,6 & 18,6 & 4,6 & 0,20 \\
\hline
\end{tabular}

Las siglas corresponden a las iniciales del nombre de la ubicación geográfica de los individuos. 


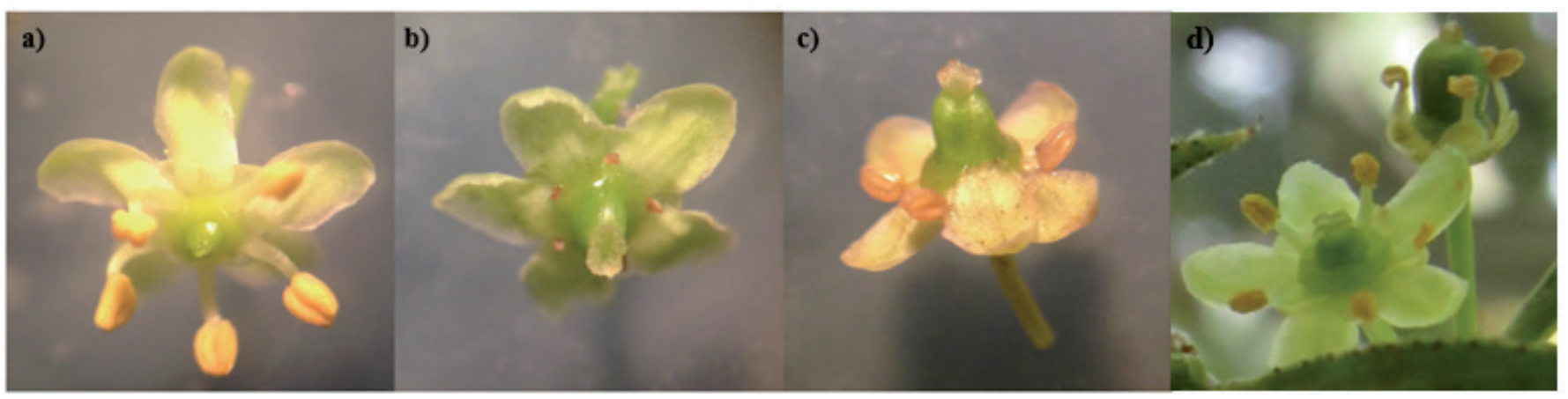

Figura 1. Tipo de flores, según sexualidad, identificadas en individuos de Maytenus boaria: a) flor masculina o estaminada con ovario atrofiado y estambres desarrollados; b) flor femenina o pistilada con ovario desarrollado y estaminodios o estambres atrofiados y c-d) flor hermafrodita o perfecta en diferentes estados de maduración de ovario y estambres, se observa el disco nectario y el estigma más o menos expuesto. / Flower types, according to sexuality, identified in individuals of Maytenus boaria: a) male or staminate flower with atrophied ovary and developed stamens; b) female or pistillate flower with developed ovary and staminodes or stunted stamens and c-d) hermaphrodite or perfect flower in different stages of ovary maturation and stamens, the nectary disc is observed and the stigma more or less exposed.

TABLA 2. Porcentaje promedio de fructificación (media \pm error estándar) en individuos predominantes femeninos y predominantes masculinos según tratamiento de polinización. Letras distintas entre los tratamientos por sexo indican diferencias significativas dentro de cada sexo predominante. / Average percentage of fructification (mean \pm standard error) in predominantly male and predominantly female individuals according to pollination treatment. Different letters between treatments by sex indicate significant differences within each predominant sex.

\begin{tabular}{lccccc}
\hline Sexo predominante & Tratamiento & $\begin{array}{c}\mathbf{N}^{\circ} \text { de } \\
\text { árboles }\end{array}$ & $\begin{array}{c}\mathbf{N}^{\circ} \text { promedio de } \\
\text { flores }\end{array}$ & $\begin{array}{c}\mathbf{N}^{\circ} \text { promedio de } \\
\text { frutos formados }\end{array}$ & $\begin{array}{c}\text { Porcentaje promedio } \\
\text { de fructificación }\end{array}$ \\
\hline Femenino & $\begin{array}{c}\text { Autopolinización } \\
\text { espontánea }\end{array}$ & 3 & $77,7 \pm 27,8$ & $0,0 \pm 0,0$ & $0,0 \pm 0,0^{\mathrm{a}}$ \\
& Polinización natural & 3 & $77,3 \pm 25,2$ & $33,0 \pm 13,3$ & $46,0 \pm 11,0^{\mathrm{b}}$ \\
\hline Masculino & $\begin{array}{c}\text { Autopolinización } \\
\text { espontánea }\end{array}$ & 6 & $147,8 \pm 42,2$ & $1,5 \pm 0,7$ & $1,2 \pm 0,9^{\mathrm{a}}$ \\
& Polinización natural & 6 & $150,2 \pm 43,8$ & $0,5 \pm 0,3$ & $0,3 \pm 0,2^{\mathrm{a}}$ \\
\hline
\end{tabular}

TABLA 3. Porcentaje promedio de antesis (media \pm error estándar) para los distintos tipos de flores tanto en individuos predominantes femeninos como predominantes masculinos. / Average percentage of anthesis (mean \pm standard error) for the different flower types in both predominantly male and predominantly female individuals.

\begin{tabular}{lcccc} 
& \multicolumn{3}{c}{ Porcentaje promedio de antesis (\%) } \\
\cline { 2 - 5 } Sexo predominante & \multicolumn{2}{c}{ Femenino } & \multicolumn{2}{c}{ Masculino } \\
\hline Fecha muestreo & Agosto & Septiembre & Agosto & Septiembre \\
Flores femeninas & $33,3 \pm 9,6$ & $79,3 \pm 5,4$ & $0,0 \pm 0,0$ & $0,0 \pm 0,0$ \\
Flores masculinas & $0,0 \pm 0,0$ & $0,0 \pm 0,0$ & $56,6 \pm 8,9$ & $87,6 \pm 3,2$ \\
Flores hermafroditas & $0,0 \pm 0,0$ & $0,0 \pm 0,0$ & $0,0 \pm 0,0$ & $6,0 \pm 2,2$ \\
\hline
\end{tabular}

\section{ARQUITECTURA}

INTERPRETACIÓN MODULAR DE LA ARQUITECTURA

El análisis de los patrones de crecimiento de $M$. boaria determinó tres unidades modulares básicas: dolicoblasto o macroblasto, inflorescencia y braquiblasto temporal reproductivo. Considerando estas tres unidades y la yema de origen se describen cuatro módulos (Fig. 2). Específicamente, dolicoblasto a partir de yema apical (módulo A) (Fig. 2), cuyo crecimiento y desarrollo puede terminar en un aborto o muerte apical (MA). Braquiblasto temporal reproductivo 
(módulo B), inflorescencia (módulo C) y dolicoblasto (módulo D), todos a partir de yema axilar (Fig. 2). Un braquiblasto temporal reproductivo corresponde a un brote de entrenudos cortos de nula o lenta elongación, el cual desarrolla flores a partir de sus yemas axilares y un dolicoblasto a partir de su yema apical (Ginocchio \& Montenegro 1992). En el caso de M. boaria, las flores que se desarrollan en los braquiblastos temporales reproductivos son solitarias, a diferencia de aquellas que se desarrollan a partir de yemas laterales de dolicoblastos, las cuales se disponen en fascículos.

En el análisis de las yemas apicales, la prueba de homogeneidad arrojó que existe dependencia entre las variables sexo predominante y tipo de módulo (Chicuadrado $=3,8679 ;$ g.l. $=1 ; p=0,0492$ ). En los dos sexos predominantes, el módulo $A$ presentó una mayor frecuencia

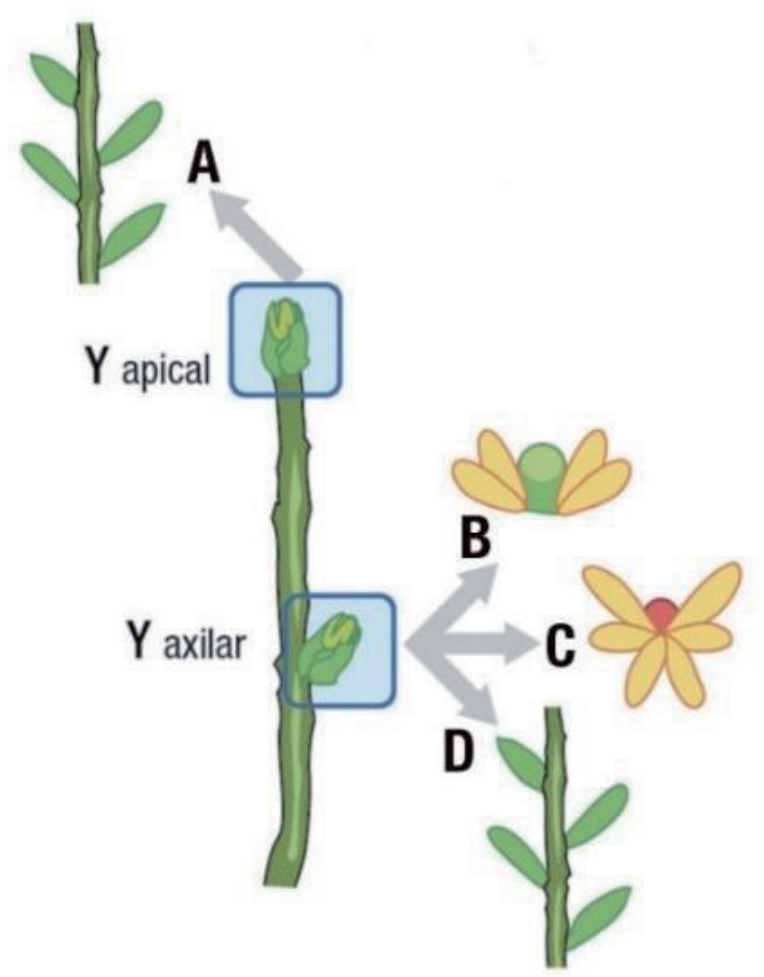

Figura 2. Tipos de módulos presentes en Maytenus boaria. Módulo $A=$ dolicoblasto, a partir de una yema apical ( $Y$ apical). Módulo $\mathrm{B}=$ braquiblasto temporal reproductivo; Módulo $C=$ inflorescencia y Módulo $\mathrm{D}=$ dolicoblasto, a partir de una yema axilar ( $Y$ axilar). / Types of modules present in Maytenus boaria. Module $A=$ dolichoblast, from an apical bud ( $Y$ apical). Module $\mathrm{B}=$ temporary reproductive brachyblast; Module $\mathrm{C}=$ inflorescence and Module $\mathrm{D}=$ dolichoblast, from an axillary bud (Y axillary). de conteo que la muerte apical, lo que constituye alrededor de un $90 \%$ de ocurrencia en ambos casos (Tabla 4). Entre individuos predominantes femeninos y predominantes masculinos no se encontraron diferencias significativas en el módulo $A(p=0,1464)$; a diferencia de la muerte apical, donde sí se presentaron diferencias significativas entre sexos $(p=$ 0,0061), siendo más frecuente en individuos predominantes femeninos (Tabla 4).

Para ambos sexos predominantes, el módulo más frecuente desarrollado a partir de yemas axilares fue el módulo $C$, seguido del módulo $B$ y finalmente del módulo D (Tabla 4). Sin embargo, las frecuencias para cada módulo fueron mayores en los individuos predominantes femeninos que en los predominantes masculinos. Específicamente, tanto el módulo $C$ como el $D$ presentaron diferencias en la frecuencia de conteo entre los sexos predominantes ( $p=0,0012$ y $p=0,0215$, respectivamente); contrario a lo ocurrido con el módulo $B$, donde no se encontraron diferencias significativas (Tabla 4).

Dentro de cada sexo predominante existieron diferencias en al menos un módulo en la frecuencia de conteo $(p=0,0026$ predominantes femeninos y $p<0,0001$ predominantes masculinos). Las diferencias significativas se observaron entre el módulo Cy $D$ para ambos sexos ( $p<0,0001$ predominantes femeninos y $p=0,0037$ predominantes masculinos); $y$ entre el módulo $B$ y $C$ en individuos predominantes femeninos ( $p$ $=0,0029)$ (Anexo 1).

\section{UNIDADES ARQUITECTURALES}

Se lograron identificar dos tipos de unidades arquitecturales principales, sin considerar una tercera unidad, la cual corresponde a una combinación de ambas, pero sin patrones específicos. La primera unidad la constituye la combinación

TABLA 4. Frecuencia de conteo de módulos por yema apical y axilar. Valores en negrita indican diferencias significativas $(p<$ 0,05 ) entre individuos predominantes femeninos y masculinos. El código de los módulos sigue la Figura 2. / Modules counting frequency per apical and axillary bud. Values in bold indicate significant differences $(p<0.05$ ) between predominantly male and female individuals. The code of the modules follows Figure 2.

\begin{tabular}{lcccc}
\hline Yema & Módulo & Femenino & Masculino & valor $p$ \\
\hline Apical & A & 19,5 & 14,5 & 0,1464 \\
& MA & 2,5 & 1,0 & 0,0061 \\
\hline Axilar & B & 112,5 & 79,5 & 0,2912 \\
& C & 243,0 & 138,0 & 0,0012 \\
& D & 69,5 & 41,0 & 0,0215 \\
\hline
\end{tabular}


de los cuatro tipos de módulos (Fig. 3). En ella, las yemas axilares de la parte basal dan origen a inflorescencias (módulo C), en la zona media a braquiblastos temporales reproductivos (módulo B) y cerca de la parte apical a dolicoblastos (módulo D). Finalmente, la yema apical de esta unidad da origen a un dolicoblasto (módulo A). La segunda unidad arquitectural solamente presenta la combinación de dos módulos: dolicoblasto en la zona apical (módulo A) e inflorescencias en yemas laterales (módulo C) (Fig. 3).

El análisis de homogeneidad mostró independencia entre las variables sexo predominante y tipo de unidad arquitectural (Chi-cuadrado $=0,285$; g.l. $=1 ; p=0,5934$ ) . Los resultados obtenidos indican que no existen diferencias significativas entre individuos predominantes femeninos y predominantes masculinos en la frecuencia de conteo de la unidad arquitectural $A(p=0,2816)$ y de la unidad arquitectural $B(p=0,1247)$ (Tabla 5). Sin embargo, existe una tendencia de los individuos predominantes masculinos a producir más unidades arquitecturales que los predominantes femeninos. Así mismo, dentro de cada sexo predominante, no se presentaron diferencias significativas en la frecuencia de conteo de ambas unidades arquitecturales $(p=0,5453$ predominante femenino y $p=0,1447$ predominante masculino) (Tabla 5). No obstante, se pudo observar una tendencia, donde la unidad arquitectural $\mathrm{A}$ presentaba una mayor frecuencia de conteo que la unidad $B$ en ambos sexos.

Tabla 5. Frecuencia de conteo de las unidades arquitecturales $A$ y $B$ en individuos predominantes femeninos y predominantes masculinos. Valor $p \geq 0,05$ indica que no existen diferencias significativas entre sexo predominante (arriba a la derecha de la tabla) y entre unidades arquitectónicas (abajo a la izquierda de la tabla). / Counting frequency of architectural units $A$ and $B$ in predominantly male and predominantly female individuals. $P$-value $\geq 0.05$ indicates that there are no significant differences between predominant sex (top right of the table) and between architectural units (bottom left of the table).

\begin{tabular}{lccc}
\hline Unidad & Femenino & Masculino & Valor $\mathbf{p}$ \\
$\mathrm{A}$ & 6,5 & 11,5 & 0,2816 \\
$\mathrm{~B}$ & 4,0 & 8,5 & 0,1247 \\
\hline Valor $\mathrm{p}$ & 0,5453 & 0,1447 & \\
\hline
\end{tabular}
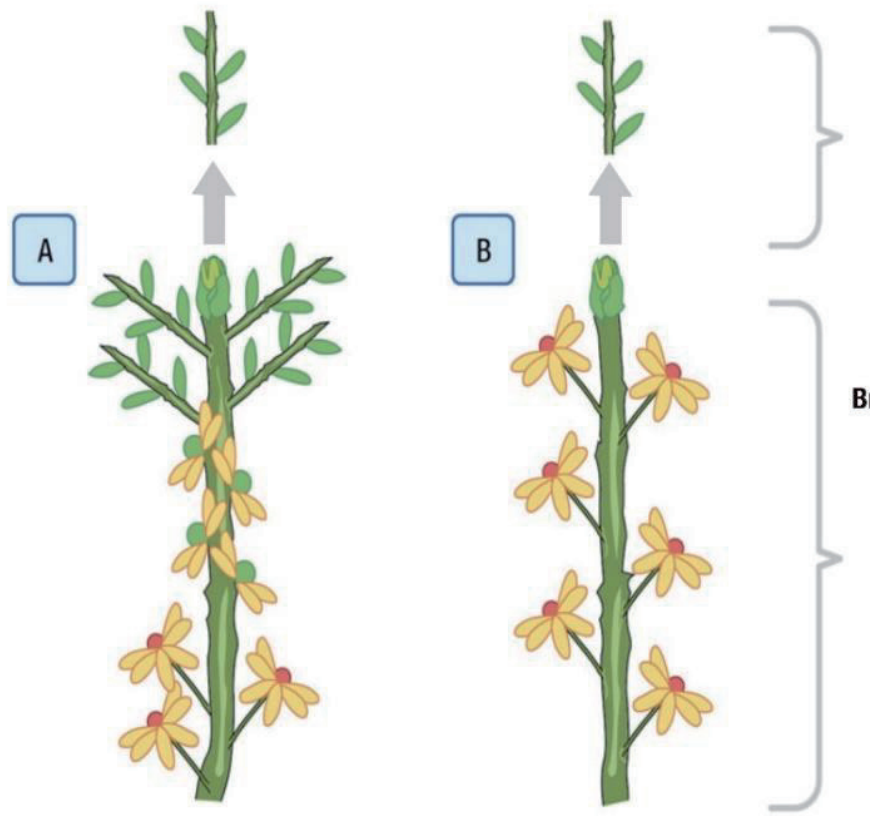

Dolicoblasto (A)

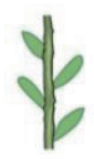

Braquiblasto temporal (B)

Inflorescencia (C)

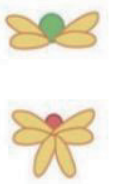

Dolicoblasto (D)

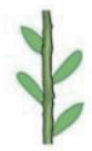

Figura 3. Tipos de unidades arquitecturales dominantes en Maytenus boaria, las cuales se forman a partir de la combinación de los distintos tipos de módulos y su distribución espacial. La unidad arquitectural A está compuesta en la yema apical por un dolicoblasto (módulo A) y en la yema axilar por un braquiblasto temporal reproductivo (módulo B), una inflorescencia (módulo C) y un dolicoblasto (módulo D). La unidad arquitectural B está compuesta en la yema apical por un dolicoblasto (módulo A) y en la yema axilar por inflorescencia (módulo C). / Type of dominant architectural units in Maytenus boaria, which are formed from the combination of the different types of modules and their spatial distribution. Architectural unit A is composed of a dolichoblast (module A) in the apical bud, a temporary reproductive brachyblast (module B), an inflorescence (module C) and a dolichoblast (module D) in the axillary bud. Architectural unit B consists of a dolicoblast in the apical bud (module A) and inflorescence in the axillary bud (module C). 


\section{Fenomorfología}

Tanto en individuos predominantes femeninos como predominantes masculinos el crecimiento vegetativo de los dolicoblastos puede ocurrir a partir de yemas apicales y laterales de dolicoblastos de la temporada anterior (desde septiembre a mediados de diciembre); así como también desde las yemas apicales de braquiblastos temporales reproductivos, entre principios de octubre y mediados de diciembre, desarrollados en la temporada pasada. Dentro de este último período se desarrollan los braquiblastos temporales reproductivos, a partir de yemas laterales de los dolicoblastos generados en la misma temporada de crecimiento (Fig. 4).

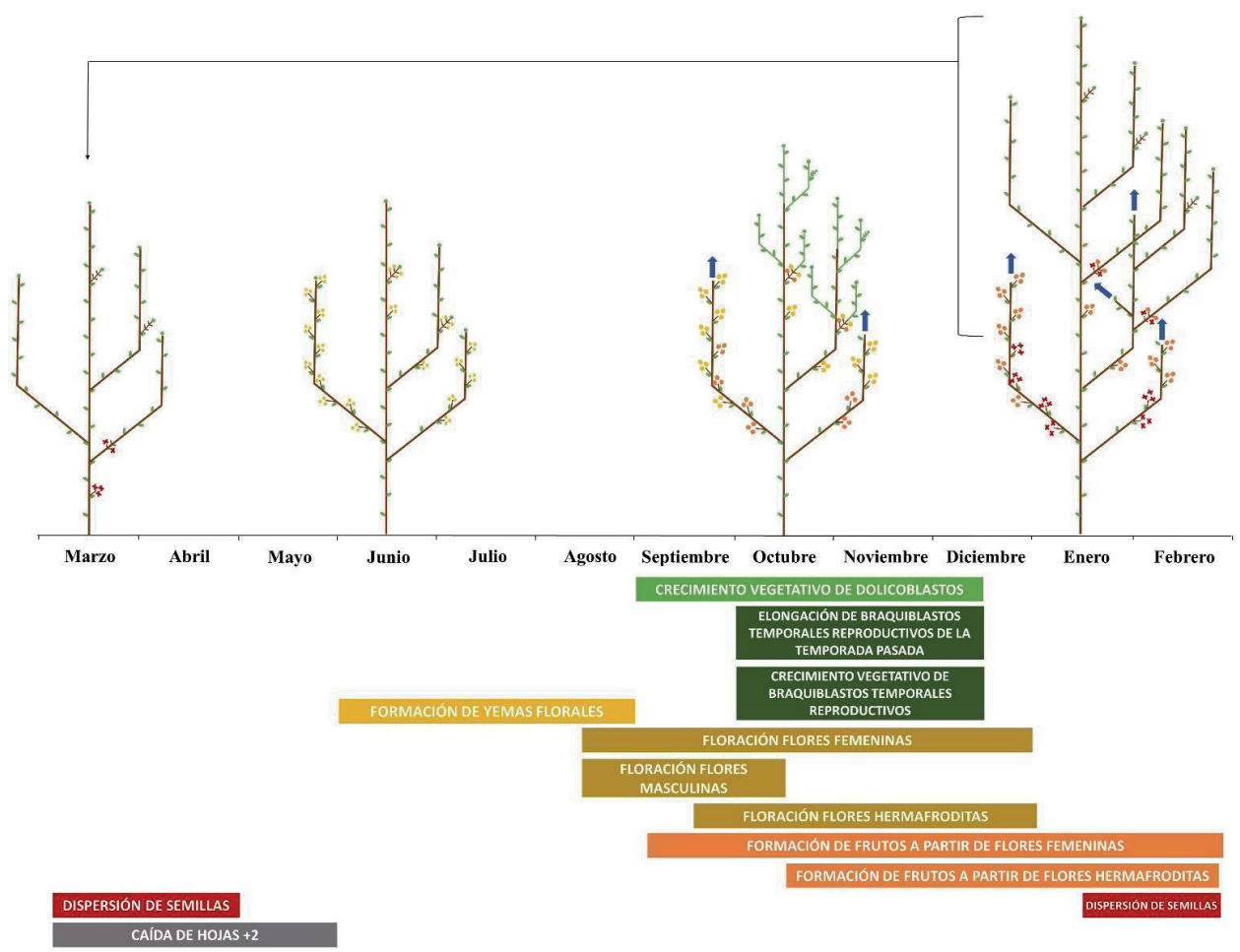

Leyenda del diagrama de la fenomorfología de Maytenus boaria Molina

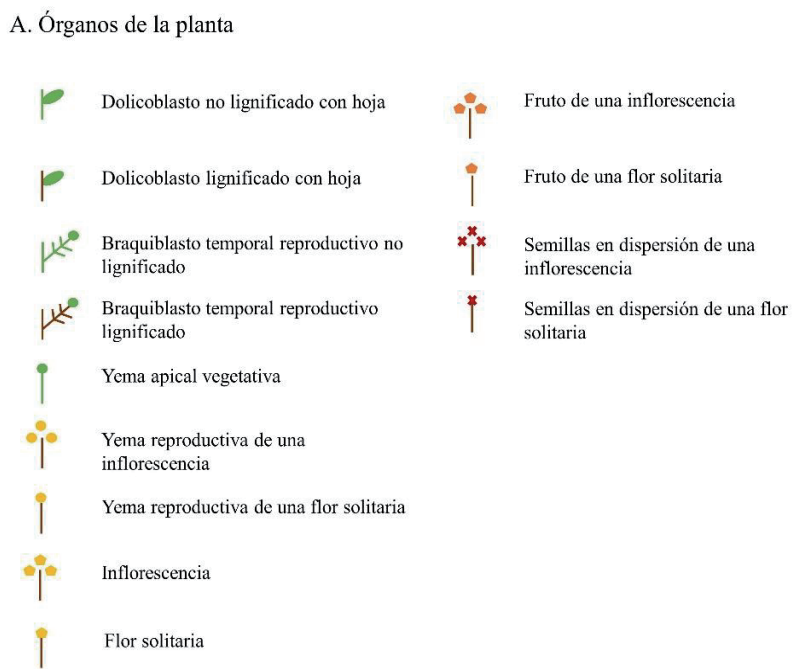

Figura 4. Diagrama fenomorfológico de Maytenus boaria (Modificado de Orshan 1989). / Phenomorphological diagram of Maytenus boaria (Modified from Orshan 1989). 
Las yemas florales se desarrollan entre junio y agosto, a partir de yemas laterales preformadas en dolicoblastos (Montenegro et al. 1989) y braquiblastos temporales reproductivos del período de crecimiento previo. La floración se desarrolla durante cinco meses y se da de forma paralela al crecimiento vegetativo, desde mediados de agosto hasta finales de diciembre. En el caso de individuos predominantes femeninos, la antesis floral tiene lugar a lo largo de los cinco meses. En el caso de individuos predominantes masculinos, las flores masculinas solo florecen entre agosto y octubre, traslapándose desde finales de septiembre y principios de octubre con la floración de las flores hermafroditas, la cual termina a finales de diciembre. La formación de frutos a partir de flores femeninas comienza unos 15 días después de la floración de estas; es decir, en septiembre y hasta finales de febrero. En cambio, la formación de frutos a partir de flores hermafroditas ocurre desde octubre hasta, probablemente, finales de febrero. La dispersión de las semillas se traslapa con la formación de frutos, comenzando a mediados de enero y finalizando en abril (Montenegro et al. 1989). La caída de hojas a partir de dolicoblastos ocurre dos años después de formadas (Montenegro et al. 1989) (Fig. 4).

\section{DISCUSIÓN}

\section{Caracterización de Individuos de Maytenus boaria}

Maytenus boaria es una de las especies nativas más frecuentes en la flora urbana de Chile central (Dobbs et al. 2011, Santilli et al. 2018). Si bien su altura en ambientes naturales puede alcanzar los 8 a 15 m (Rodríguez et al. 1983), en estudios realizados en la ciudad de Santiago, los valores fluctuaron entre 4 y $6 \mathrm{~m}$ y los de diámetro a la altura del pecho (DAP) entre 8 y $21 \mathrm{~cm}$ (Dobbs et al. 2011). Estos valores son muy similares a los registrados en los individuos seleccionados en el presente estudio (Tabla 1), probablemente debido a que están influenciados por similares condiciones de sitio y prácticas de manejo urbano (Dobbs et al. 2011). La copa de los individuos seleccionados fue simétrica, ya que existió similitud en el promedio de los dos ejes medidos de la copa (Tabla 1). Lo cual, indicaría una homogeneidad de copa, similar a lo descrito por Dobbs (2005) para M. boaria.

\section{Aspectos REPRODUCTIVOS Y SISTEMA SEXUAL}

El análisis del sistema sexual demuestra que $M$. boaria es una especie dioica y andromonoica. La dioecia ha sido reportada para esta especie por diversos autores (Donoso \& Wendler 1985, Arroyo \& Uslar 1993, Barrera \& Meza 2006, Dawson 2016). Sin embargo, Hoffmann et al. (2003) y Hoffmann (2005) son los únicos autores que la describen como poligamomonoica, aunque señalando una condición trimonoica (presencia de flores hermafroditas, masculinas y femeninas en un mismo pie). La poca claridad en la caracterización del sistema sexual de M. boaria se repite no solo en especies del género Maytenus, como por ejemplo en Maytenus magellanica (Rodríguez et al. 1983, Arroyo \& Squeo 1990, Riedemann \& Aldunate 2001, Hoffmann 2005, Barrera \& Meza 2006), sino que también en especies de la familia Celastraceae (Brizicky 1964, Sebsebe 1985, Matthews \& Endress 2005). Benevides et al. (2013) postulan que esto puede tener relación con el tamaño pequeño de las flores y las modificaciones morfo-anatómicas de las estructuras femeninas y masculinas de las especies del género. Sin embargo, Donoso \& Wendler (1985) indican que el polimorfismo tendría su origen en la plasticidad que presenta la especie.

En cuanto al análisis de los aspectos reproductivos de M. boaria, los bajos porcentajes de fructificación por autopolinización espontánea indicarían que la especie no tiene una alta autogamia o autofertilización autónoma. La ausencia de fructificación observada para el tratamiento de autopolinización espontánea en los individuos predominantes femeninos indica que esta especie no es apomíctica, y por tanto necesitaría de polen cruzado proveniente de individuos predominantes masculinos para la producción de semillas. Así, se sugiere la necesidad de un vector de polinización para el transporte de polen hacia las flores femeninas. Esta especie tendría un carácter entomófilo (Judd et al. 1999), ya que ha sido clasificada como una especie melífera o de interés apícola (Montenegro et al. 1992, Forcone \& Kutschker 2006). En este estudio no se tomaron datos de tasas de visitas de polinizadores, pero se observaron al pehuenche negro (Elaphroptera nigripennis (Smith)), la abeja melífera (Apis mellifera L.) y dípteros no identificados libando frecuentemente las flores de $M$. boaria; estos insectos probablemente actúen como agentes polinizadores. Con todos estos antecedentes, M. boaria podría considerarse una especie xenógama.

La fructificación observada en los árboles predominantes masculinos corroboraría la presencia de flores hermafroditas, en concordancia con el carácter bisexual descrito para las especies del género Maytenus (Hou 1955, Navas 1976). Dado que se observaron dos morfos florales para las flores hermafroditas (Fig. 1), se puede presumir que parte de los frutos se formaron a partir del morfo floral donde las anteras se disponen a la misma altura del estigma, lo cual promovería la autofecundación (Tébar \& Llorens 1997). Por otro lado, los bajos porcentajes de fructificación se pueden explicar por la existencia de un morfo floral que presenta sus anteras por debajo del estigma, lo cual previene la autopolinización 
(Anderson \& Symon 1989, Barrett 2002, Barrett 2010). Si bien en este estudio se visualizó la formación de frutos a partir de flores hermafroditas, estos no lograron desarrollarse, lo que se explicaría por abortos selectivos de los frutos (Sutherland \& Delph 1984, Knight et al. 2005). De acuerdo con Bawa \& Webb (1984), el aborto de frutos puede influenciar el éxito reproductivo de la función femenina y masculina de las flores hermafroditas, teniendo en consideración que el gradiente de variación morfológica de estas flores puede repercutir en la funcionalidad sexual de las mismas (Benevides et al. 2013). Por tanto, se presume que la mayoría de las flores perfectas que presenta $M$. boaria son morfológicamente hermafroditas, pero fisiológicamente masculinas. Bajo este contexto, la andromonoecia se presenta principalmente como una estrategia espacial y temporal para remover y depositar polen (Primack \& Lloyd 1980), lo cual ha sido planteado por algunos autores como pasos intermedios del hermafroditismo a la dioecia (Barrett 2002, Barrett 2010, Spigler \& Ashman 2012).

En el análisis del sistema sexual, los individuos andromonoicos presentaron una gran variabilidad en la proporción de flores hermafroditas (1,25 a 14,97\%). Tanto Diggle (1993) como Emms (1993) han indicado que la expresión sexual en especies andromonoicas ha demostrado ser ampliamente versátil entre individuos de una misma población. Ello estaría dado por una alta influencia de factores medioambientales, como la temperatura (Nakasone \& Paull 1998), disponibilidad de nutrientes (Primack \& Lloyd 1980) y estatus hídrico (Tébar \& Llorens 1997). Probablemente la discrepancia que existe entre los distintos autores sobre el sistema sexual de M. boaria se deba a esta variación. Sunnichan et al. (2004) reportaron en Sterculia urens Roxb. (especie andromonoica) una variación anual en la presencia de flores hermafroditas, donde algunos individuos incluso nunca las presentaron. Esto lleva a suponer que M. boaria podría presentar variaciones periódicas en la proporción de flores hermafroditas de cada individuo y/o población, lo cual explicaría que haya sido caracterizada como una especie dioica.

\section{ARQUITECTURA}

En este estudio, Maytenus boaria presentó tres de las cinco unidades morfológicas básicas descritas para las especies leñosas que dominan la zona mediterránea de Chile central, las cuales pueden desarrollarse a partir de yemas apicales o axilares (Ginocchio \& Montenegro 1992). Estas tres unidades se representan en cuatro módulos que consideran la yema de origen: dolicoblasto de yema apical (módulo A), dolicoblasto de yema axilar (módulo D) e inflorescencias de yema axilar (módulo C) (Fig. 2), descritos en estudios anteriores (Montenegro et al. 1989, Ginocchio \& Montenegro 1992), y braquiblasto temporal reproductivo de yema axilar (módulo B). Este último, probablemente descrito por Lourteig (1988) como brácteas en ramitas acortadas. En M. boaria se observó que este braquiblasto puede alargarse levemente o bien generar un dolicoblasto en su yema apical, lo cual le permitiría un rápido reemplazo del área foliar con baja inversión de recursos, ya que éstos evitarían la formación de entrenudos (Ginocchio \& Montenegro 1992, Ginocchio \& Montenegro 1994). Además, este órgano podría tener una estrategia reproductiva, considerando que en M. boaria son capaces de producir flores.

En el crecimiento vegetativo de $M$. boaria están involucrados los braquiblastos temporales reproductivos y los dolicoblastos. Los dolicoblastos de yema apical serían los principales responsables del crecimiento vertical en altura (Wang et al. 2018). En cambio, los provenientes de las yemas axilares, y predominantes en las especies perennifolias de la zona mediterránea de Chile central (Ginocchio \& Montenegro 1994), permitirían un amplio rango de estratificación vertical (Ginocchio \& Montenegro 1994, Wang et al. 2018). Por otro lado, el aborto en baja proporción de los dolicoblastos provoca un crecimiento definido, lo cual modelaría la arquitectura simpodial que presenta la especie (Montenegro et al. 1989, Perreta \& Vegetti 2005). Esta sería la única estrategia de $M$. boaria para limitar el crecimiento vegetativo si se considera que no desarrolla inflorescencias en las yemas apicales (Herrera 1987, Ginocchio \& Montenegro 1992). Contrario a lo que ocurre en la mayoría de las especies siempreverdes del matorral chileno, donde predominan las inflorescencias tanto en las yemas axilares como apicales (Ginocchio \& Montenegro 1992).

Tanto en individuos femeninos como masculinos, la reproducción está dada mayoritariamente por las inflorescencias; secundariamente por los braquiblastos temporales reproductivos (Fig. 4). En el caso de los braquiblastos, las flores se disponen solitarias en las yemas axilares (Lourteig 1988), mientras que en las inflorescencias van en fascículos (Montenegro 2000, Dawson 2016). Probablemente, la reproducción de flores solitarias del braquiblasto temporal reproductivo tenga una estrategia temporal más que cuantitativa. La mayor cantidad de inflorescencias observadas en los individuos femeninos que en los masculinos podría responder a un mecanismo de compensación, ya que las flores fasciculadas femeninas (tres/fascículo) están dispuestas en menor cantidad que las masculinas (ocho a diez/fascículo) (Rodríguez et al. 1983, Lourteig 1988, Riedemann \& Aldunate 2001). Por lo tanto, y según lo evidenciado en los resultados de biología reproductiva (Tabla 2), la producción de flores en individuos 
femeninos es menor que en los masculinos, lo cual le permitiría ahorrar energía y derivarla a la formación de frutos (Maillette 1992).

Por otro lado, los individuos femeninos de M. boaria tienden a crecer con menos unidades arquitecturales, pero con más módulos, a diferencia de los masculinos que desarrollan una tendencia inversa. Esta podría ser una estrategia de los individuos femeninos para evitar el costo de producir más unidades para sustentar un mayor número de módulos. Sin embargo, esta condición sería variable ya que la producción de módulos responde a factores ambientales (Montserrat-Martí et al. 2004). Por ejemplo, después de incendios y herbivoría, no hay variación en el tipo de módulo, sino en la ocurrencia de diferentes proporciones y su disposición espacial (Ginocchio \& Montenegro 1994, Ginocchio et al. 1994).

La arquitectura descrita para M. boaria, probablemente sea específica para las condiciones presentes en el área de estudio. Esta arquitectura particular y sus patrones de ramificación tienen ventajas en ciertos hábitats (Küppers 1989). Por ello, se esperaría que cambie durante el proceso de crecimiento y en respuesta a las condiciones ambientales donde se desarrolla (Ginocchio et al. 1994, Wang et al. 2018). Estas variaciones pueden estar representadas en el polimorfismo que presenta la especie debido a su plasticidad (Donoso \& Wendler 1985). Es recomendable investigar la arquitectura de M. boaria en un gradiente latitudinal y/o altitudinal, para conocer la variación de dicha arquitectura a través de su amplia distribución geográfica (Donoso \& Wendler 1985).

\section{Fenomorfología}

Según los resultados de este estudio, Maytenus boaria tiene una estrategia fenológica donde las fenofases se traslapan en un corto período de tiempo, especialmente el crecimiento vegetativo con el proceso reproductivo (Montserrat-Martí et al. 2004). Esta sería una habilidad para aprovechar el corto período de crecimiento favorable, característico de las regiones de clima mediterráneo (Ginocchio \& Montenegro 1996).

Contrario a lo que ocurre en la mayoría de las especies leñosas del matorral, M. boaria sigue un patrón donde la floración antecede al crecimiento vegetativo (Montenegro et al. 1988, 1989). Esto ocurriría por la arquitectura que posee la especie, ya que al igual que en Peumus boldus Molina, Colliguaja odorifera Molina y Sophora macrocarpa Sm., las yemas florales se diferencian sobre los dolicoblastos del año anterior. Lo mismo ocurriría con las yemas florales en los braquiblastos temporales reproductivos, ya que al florecer el braquiblasto tiene una temporada de crecimiento (Ginocchio
\& Montenegro 1992). Según estudios anteriores, el período de floración de M. boaria va desde septiembre a mediados de diciembre (Montenegro et al. 1989). Sin embargo, la iniciación en este estudio se registró a mediados de agosto, acumulando cinco meses de floración en los individuos femeninos y andromonoicos, y dos meses en los masculinos. En el caso de los individuos andromonoicos existe una floración asincrónica, ya que las flores unisexuales abren antes y por un periodo más corto ( 2 meses) que las hermafroditas (3,5 meses), traslapándose entre ellas en un corto periodo. Este traslape se debería a que las flores masculinas se encuentran en estado de senescencia, y la única fuente de polen para la fecundación de las flores femeninas provendría de las flores hermafroditas, cuyo rol sería masculino. En los individuos femeninos y andromonoicos de M. boaria pareciera estar predominando un patrón de floración extendida, ya que ésta estaría asociada a especies autoincompatibles, que producen flores en forma constante con pocas flores asincrónicas en un periodo prolongado (Augspurger 1983, De Jong et al. 1992). Esta estrategia fenológica de M. boaria difiere de la descrita en poblaciones australes de Argentina, donde la duración de la floración es más corta (2,5 meses), extendiéndose desde final de invierno hasta mediados de primavera (Lediuk et al. 2014). En Nueva Zelanda, donde M. boaria es considerada maleza, la floración solo dura 1,5 meses y se extiende desde final de agosto hasta principio de octubre (Dawson 2016).

El crecimiento vegetativo de los dolicoblastos a partir de las yemas de renuevo preformadas la temporada pasada (Montenegro et al. 2006) se inicia un mes antes que el crecimiento del braquiblasto temporal reproductivo. Este retraso podría ocurrir debido al inicio de la floración del braquiblasto. Las especies de hoja perenne que desarrollan dolicoblastos como braquiblastos temporales tienen una mayor plasticidad bajo las condiciones climáticas mediterráneas, ya que al eliminar parte de sus hojas de dolicoblasto en verano reducen la zona de transpiración (Ginocchio \& Montenegro 1994). En estas especies, a diferencia de las deciduas de verano, los braquiblastos temporales se forman a lo largo de la estación de crecimiento (Ginocchio \& Montenegro 1992).

La cantidad de dolicoblastos desarrollados la temporada anterior no solo tiene relevancia en el crecimiento vegetativo de la especie, sino también en la producción anual de frutos, ya que las inflorescencias se forman a partir de las yemas axilares presentes en ellos (Montserrat-Martí et al. 2004). La formación de frutos se inicia primero desde las flores femeninas y secundariamente desde las hermafroditas, lo cual es esperable considerando que está directamente relacionado con la época de floración (Fig. 4). La formación de frutos de flores femeninas comienza 15 días después de 
la floración de éstas (septiembre y hasta finales de febrero) y se extiende por casi seis meses, lo cual estaría relacionado al tamaño del fruto (Castro-Díez \& Montserrat-Martí 1998) y a la elaboración de gran cantidad de ácidos grasos en las semillas (Menegoz \& Zapata 2017). La dispersión de las semillas se traslapa con la formación de frutos, comenzando a mediados de enero y finalizando en abril (Montenegro et al. 1989). El estudio de la fenología en otras áreas de distribución sería interesante, no solo para diseñar un adecuado manejo productivo de la especie, sino también para entender cómo la variación climática podría estar afectando en la duración de las fenofases.

\section{AGRADECIMIENTOS}

Los autores agradecen el financiamiento de ANID PIA/ BASAL FB0002 y a Mauricio Caroca por su ayuda con los análisis estadísticos. Los autores agradecen también los comentarios y los aportes de los revisores del presente artículo, ya que fueron valiosos para la generación de esta versión final del artículo.

\section{REFERENCIAS}

Anderson, G.J., Symon, D.E. 1989. Functional dioecy and andromonoecy in Solanum. Evolution 43(1): 204-219.

Arroyo, M.T.K., Squeo, F.A. 1990. Relationship between plant breeding systems and pollination. In: Kawano, S. (Ed.), Biological approaches and evolutionary trends in plants, pp. 205-227. Academic Press, London, United Kingdom.

Arroyo, M.T.K., Uslar, P. 1993. Breeding system in a temperate Mediterranean-type climate montane sclerophyllous forest in central Chile. Botanical Journal of the Linnean Society 111(1): 83-102.

Augspurger, C.K. 1983. Phenology, flowering synchrony, and fruit set of six neotropical shrubs. Biotropica 15(4): 257267.

Barrera, E., Meza, I. 2006. Epidermis foliar de árboles chilenos. Museo Nacional de Historia Natural Chile. Publicación ocasional $\mathrm{N}^{\circ}$ 60: 5-90.

Barrett, S.C.H. 2002. Evolution of sex: the evolution of plant sexual diversity. Nature Reviews Genetics 3(4): 274-284.

Barrett, S.C.H. 2003. Mating strategies in flowering plants: the outcrossing-selfing paradigm and beyond. Philosophical Transactions of the Royal Society B Biological Sciences 358(1434): 991-1004.

Barrett, S.C.H. 2010. Understanding plant reproductive diversity. Philosophical Transactions of the Royal Society B:
Biological Sciences 365(1537): 99-109.

Bawa, K.S., Webb, C.J. 1984. Flower, fruit and seed abortion in tropical forest trees: implications for the evolution of paternal and maternal reproductive patterns. American Journal of Botany 71(5): 736-751.

Benevides, C.R., Haddad, I.V.N., Barreira, N.P., Rodarte, A.T.A., Galetto, L., Santiago-Fernandes, L.D.R., Lima, H.A. 2013. Maytenus obtusifolia Mart. (Celastraceae): a tropical woody species in a transitional evolutionary stage of the gynodioecy-dioecy pathway. Plant Systematics and Evolution 299(9): 1693-1707.

Biral, L., Simmons, M.P., Smidt, E.C., Tembrock, L.R., Bolson, M., Archer, R.H., Lombardi, J.A. 2017. Systematics of new world Maytenus (Celastraceae) and a new delimitation of de genus. Systematic Botany 42 (4): 680-693.

Brizicky, G. K. 1964. The genera of Celastrales in the southeastern United States. Journal of the Arnold Arboretum 45(2): 206-234.

Castro-Díez, P., Montserrat-Martí, G. 1998. Phenological pattern of fifteen Mediterranean phanaerophytes from Quercus ilex communities of NE-Spain. Plant Ecology 139(1): 103112.

Cuartas-Domínguez, M., Rojas-Céspedes, A., Jara-Arancio, P., Arroyo, M. T. K. 2017. Sistema reproductivo de Trichopetalum plumosum (Ruiz \& Pav.) J. F. Macbr. (Asparagaceae), geófita endémica de Chile. Gayana Botánica 74(1): 73-81.

Dawson, M. 2016. Chilean mayten (Maytenus boaria) a ticking time bomb? New Zealand Garden Journal 19(2): 28-32.

De Jong, T.J., Klinkhamer, P.G.L., Van Staalduinen, M.J. 1992. The consequences of pollination biology for selection of mass or extended blooming. Functional Ecology 6(5): 606-615.

Di Castri, F., Hajek, E. 1976. Bioclimatología de Chile. Vicerrectoría Académica de la Universidad Católica de Chile, Santiago. $128 \mathrm{pp}$.

Di Rienzo, J.A., Casanoves, F., Balzarini, M.G., González, L., Tablada, M., Robledo, C.W. 2016. InfoStat, versión 2016. Grupo InfoStat, FCA, Universidad Nacional de Córdoba, Argentina.

Diggle, P.K. 1993. Developmental plasticity, genetic variation and the evolution of andromonoecy in Solanum hirtum (Solanaceae). American Journal of Botany 80(8): 967-973.

Dobbs, C. 2005. Construcción de modelos de estimación de biomasa y área foliar para diez especies arbóreas urbanas de la ciudad de Santiago. Tesis Ingeniería Forestal. Facultad de Ciencias Forestales, Universidad de Chile. Santiago, Chile. 79 pp.

Dobbs, C., Hernández, J., Escobedo, F. 2011. Above ground biomass and leaf area models based on a non destructive method for urban trees of two communes in Central 
Chile. Bosque 32(3): 287-296.

Donoso, C., Wendler, J. 1985. Antecedentes morfológicos y genecológicos de Maytenus boaria. Bosque 6(2): 93-99.

Donoso, C., Cabello, A., Escobar, B. 2006. Maytenus boaria Mol., Maitén, Celastraceae. En: Donoso, C. (Ed.), Las especies arbóreas de los bosques templados de Chile y Argentina. Autoecología, pp. 365-374. Marisa Cuneo Ediciones, Valdivia, Chile.

Dytham, C. 2011. Choosing and Using Statistics: A Biologist's Guide. Blackwell Science, Oxford, United Kingdom. 298 pp.

Eckert, C.G., Kalisz, S., Geber, M.A., Sargent, R., Elle, E., Cheptou, P. O., Goodwillie, C., Johnston, M.O., Kelly, J.K., Moeller, D.A., Porcher, E., Ree, R.H., Vallejo-Marín, M., Winn, A.A. 2010. Plant mating systems in a changing world. Trends in Ecology and Evolution 25(1): 35-43.

Emms, S.K. 1993. Andromonoecy in Zigadenus paniculatus (Liliaceae): spatial and temporal patterns of sex allocation. American Journal of Botany 80(8): 914-323.

Forcone, A., Kutschker, A. 2006. Floración de las especies de interés apícola en el noroeste de Chubut, Argentina. Revista del Museo Argentino de Ciencias Naturales nueva serie 8(2): 151-157.

Ginocchio, R., Montenegro, G. 1992. Interpretation of metameric architecture in dominant shrubs of the Chilean matorral. Oecologia 90(3): 451-456.

Ginocchio, R., Montenegro, G. 1994. Effects of insect herbivory on plant architecture. In: Arianoutsou, M., Groves, R.H. (Eds.), Plant-animal interactions in mediterranean-type ecosystems, pp. 115-122. Kluwer Academic Publishers, The Netherlands.

Ginocchio, R., Montenegro, G. 1996. On the structural organization of the renewal buds and their implication for the survival of embryonic structures in central Chilean matorral shrubs. Revista Chilena de Historia Natural 69: 171-181.

Ginocchio, R., Holmgren, M., Montenegro, G. 1994. Effect of fire on plant architecture in Chilean shrubs. Revista Chilena de Historia Natural 67(2): 177-182.

Gusinde, M. 1917. Medicina e higiene de los antiguos araucanos. Publicaciones del Museo de etnología y antropología de Chile (Tomo I) 4(3): 177-293.

Herrera, J. 1987. Biología reproductiva de algunas especies del matorral de Doñana. Anales del Jardín Botánico de Madrid 44(2): 483-497.

Hoffmann, A. 2005. Flora silvestre de Chile, zona Araucana. Quinta edición. Fundación Claudio Gay. Santiago, Chile. 275 pp.

Hoffmann, A., Kummerow, J. 1962. Aspectos anatómicos, morfológicos y de la fisiología de germinación de semillas de Maytenus boaria Mol. Phyton (Argentina) 18: 51-6.

Hoffmann, A., Farga, C., Lastra, J., Veghazi, E. 2003. Plantas medicinales de uso común en Chile. Tercera edición. Fundación Claudio Gay. Santiago, Chile. 275 pp.

Hou, D. 1955. A revision of the genus Celastrus. Annals of the Missouri Botanical Garden 42(3): 215-302.

Judd, W.S., Campbell, C.S., Kellogg, E.A., Stevens, P.F. 1999. Plant systematics: A phylogenetic approach. Sinauer Associates, Massachusetts. 464 pp.

Knight, T.M., Steets, J.A., Vamosi, J.C., Mazer, S.J., Burd, M., Campbel, D.R., Dudash, M.M.R., Johnston, M.O., Mitchell, R.J., Ashman, T.L. 2005. Pollen limitation of plant reproduction: patterns and process. Annual Review of Ecology, Evolution, and Systematics 36: 467-497.

Küppers, M. 1989. Ecological significance of above-ground architectural patterns in woody plants: a question of costbenefit relationships. Trends in Ecology \& Evolution 4(12): 375-379.

Larson, B.M.H., Barrett, S.C.H. 2000. A comparative analysis of pollen limitation in flowering plants. Biological Journal of the Linnean Society 69(4): 503-520.

Lediuk, K., Damascos, M.A., Puntieri, J.G., Svriz, M. 2014. Differences in phenology and fruit characteristic between invasive and native woody species favor exotic species invasiveness. Plant Ecology 215(12): 1455-1467.

Lloyd, D.G., Schoen, D.J. 1992. Self- and cross-fertilization in plants. I. Functional dimensions. International Journal of Plant Sciences 153(3): 358-369.

Loesener, T. 1942. Celastraceae. In: Engler, A., Harms, H., Mattfeld, J. (Eds.), Die natürlichen pflanzenfamilien, Vol. 20b, pp. 87-197. Duncker \& Humblot, Berlin.

Lourteig, A. 1988. Celastraceae. En: Correa, M.N. (Ed.), Flora Patagónica, Tomo VIII, Parte V, dicotiledóneas dialipétalas, pp. 103-109. INTA, Buenos Aires, Argentina.

Luebert, F., Pliscoff, P. 2017. Sinopsis bioclimática y vegetacional de Chile. Editorial Universitaria, segunda edición. Santiago, Chile. 381 pp.

Maillette, L. 1992. Seasonal model of modular growth in plants. Journal of Ecology 80(1): 123-130.

Matthews, M.L., Endress, P.K. 2005. Comparative floral structure and systematics in Celastrales (Celastraceae, Parnassiaceae, Lepidobotryaceae). Botanical Journal of the Linnean Society 149(2): 129-194.

Menegoz, K., Zapata, A. 2017. Flora cordillerana del Ñuble y sus usos tradicionales. Consejo Nacional de la Cultura y de las Artes, Chillán, Chile. 92 pp.

Meusel, H., Jäger, E., Moerchen, G. 1977. The study of growthforms of high plants in relation to application of biocides. In: Miyawaki, A., Tuxen, R. (Eds.), Vegetation Science and Environmental Protection, pp. 71-76. International 
Symposium on Protection of the Environment, Tokyo.

Montenegro, G. 2000. Chile nuestra flora útil. Ediciones Universidad Católica de Chile. Santiago, Chile. 267 pp.

Montenegro, G., Gómez, M., Ávila, G. 1992. Importancia relativa de especies cuyo polen es utilizado por Apis mellifera en el área de la Reserva Nacional Los Ruiles, VII Región de Chile. Acta Botánica Malacitana 17: 167-174.

Montenegro, G., Aljaro, M., Ávila, G. \& Mujica, A. 1988. Growth patterns as determined by water stress and adaptation. Laboratorio de Botánica, Facultad de Ciencias Biológicas, Pontificia Universidad Católica de Chile. Santiago, Chile. $9 \mathrm{pp}$.

Montenegro, G., Gómez, M., Mujica, A.M., Díaz, J. 2006. Training course on: MAPs (Medicinal and Aromatic Plants) conservation trough sustainable harvesting. Pontificia Universidad Católica de Chile, Facultad de Agronomía e Ingeniería Forestal, Programa de Magíster en Recursos Naturales. Santiago, Chile. 45 pp.

Montenegro, G., Ávila, G., Aljaro, M.E., Osorio, R., Gómez, M. 1989. Description of plant annual cycles: Chile. In: Orshan, G. (Ed.), Plant pheno-morphological studies in Mediterranean type ecosystems, pp. 347-387. Kluwer Academic Publishers, Dordrecht, The Netherlands.

Montserrat-Martí, G., Palacio-Blasco, S., Milla-Gutiérrez, R. 2004. Fenología y características funcionales de las plantas leñosas mediterráneas. En: Valladares, F. (Ed.), Ecología del bosque mediterráneo en un mundo cambiante, pp. 129-162. Ministerio de Medio Ambiente, Madrid, España.

Muñoz, C. 1966. Sinopsis de la flora silvestre de Chile. Editorial Universidad de Chile, segunda edición. Santiago, Chile. $500 \mathrm{pp}$.

Nakasone, H.Y, Paull, R.E. 1998. Tropical fruits. In: Atherton, J. (Ed.), Crop production science, Horticulture series, pp. 173-207. CAB International, New York, USA.

Navas, L.E. 1976. Flora de la cuenca de Santiago de Chile. Tomo II. Editorial Universidad de Chile. Santiago, Chile. 559 pp.

Orshan, G. 1989. Introduction. In: Orshan, G. (Ed.), Plant phenomorphological studies in Mediterranean type ecosystems, pp. 1-5. Kluwer Academic Publishers, Dordrecht, The Netherlands.

Perreta, M.G., Vegetti, A.C. 2005. Patrones estructurales en las plantas vasculares: una revisión. Gayana Botánica 62(1): 9-19.

Pirani, J.R., De Carvalho-Okano, R.M. 1999. Maytenus rupestris (Celastraceae), a new species from Minas Gerais, southeastern Brazil. Novon 9(1): 95-97.

Primack, R.B. 1985. Longevity of individual flowers. Annual Review of Ecology and Systematics 16(1): 15-37.

Primack, R.B., Lloyd, D.G. 1980. Andromonoecy in the New Zealand montane shrub manuka, Leptospermum scoparium
(Myrtaceae). American Journal of Botany 67(3): 361-368.

R Development Core Team. 2010. R: A language and environment for statistical computing. R Foundation for Statistical Computing, Vienna, Austria. URL: http://www.R-project. org.

Riedemann, P., Aldunate, G. 2001. Flora nativa de valor ornamental. Identificación y propagación. Chile zona centro. Editorial Andrés Bello, Santiago, Chile. 566 pp.

Rivera-Hutinel, A., Acevedo-Orellana, F. 2017. Biología floral y reproductiva de Escallonia pulverulenta (Ruiz et Pav.) Pers. (Escalloniaceae) y su relación con los visitantes florales. Gayana Botánica 74(1): 82-93.

Rodríguez, R., Matthei, S., Quezada, M. 1983. Flora arbórea de Chile. Tercera edición. Ediciones Universidad de Concepción, Concepción, Chile. 408 pp.

Rodríguez, R., Marticorena, C., Alarcón, D., Baeza, C., Cavieres, L., Finot, V.L., Fuentes, N., Kiessling, A., Milhoc, M., Pauchard, A., Ruiz, E., Sánchez, P., Marticorena, A. 2018. Catálogo de las plantas vasculares de Chile. Gayana Botánica 75(1): 1-430.

Santilli, L., Castro, S.A., Figueroa, J.A., Guerrero, N., Ray, C., Romero-Mieres, M., Rojas, G., Lavandero, N. 2018. Exotic species predominates in the urban woody flora of central Chile. Gayana Botánica 75(2): 568-588.

Santos, V.L.D., Costa, V.B.M., Agra, M.D.F., Silva, B.A.D., Batista, L.M. 2007. Pharmacological studies of ethanolic extracts of Maytenus rigida Mart. (Celastraceae) in animal models. Revista Brasileira de Farmacognosia 17(3): 336-342.

Sebsebe, D. 1985. The genus Maytenus (Celastraceae) in NE tropical Africa and tropical Arabia. Symbolae Botanicea Upsaliensis 25: 1-101.

Spigler, R.B., Ashman, T.L. 2012. Gynodioecy to dioecy: are we there yet? Annals of Botany 109(3): 531-543.

Sunnichan, V.G., Ram, H.M., Shivanna, K.R. 2004. Floral sexuality and breeding system in gum karaya tree, Sterculia urens. Plant Systematics and Evolution 244(3-4): 201-218.

Sutherland, S., Delph, L.F. 1984. On the importance of male fitness in plants: patterns of fruit-set. Ecology 65(4): 1093-1104.

Tébar, F.J., Llorens, L. 1997. Floral biology of Cneorum tricoccon L. (Cneoraceae): an unknown case of andromonoecy. Collectanea Botanica 23: 105-113.

Tinto, J. 1977. Utilización de los recursos forestales argentinos. Folleto técnico forestal 41. Instituto Forestal Nacional. Subsecretaría de Recursos Naturales Renovables y Ecología, Ministerio de Economía, Secretaría de Estado de Agricultura y Ganadería, Buenos Aires, Argentina. 117 pp.

Tourn, G.M., Barthélémy, D., Grosfeld, J. 1999. Una aproximación a la arquitectura vegetal: conceptos, objetivos y metodología. Boletín de la Sociedad Argentina de 
Botánica 34(1-2): 85-99.

Wang, B., Smith, S.M., Li, J. 2018. Genetic regulation of shoot architecture. Annual Review of Plant Biology 69: 437468.

Zapata, N., Budia, F., Silva, G., Viñuela, E., Medina, P. 2006. Actividad antialimentaria de Maytenus boaria Mol., Peumus boldus Mol. y Quillaja saponaria Mol. sobre
Spodoptera littoralis (Boisd.). Boletín de Sanidad Vegetal. Plagas 32(1): 125-135.

Zimmerman, M. 1988. Nectar production, flowering phenology, and strategies for pollination. In: Lovett, J., Lovett, L. (Eds.), Plant reproductive ecology: patterns and strategies, Vol. 41, pp. 157-178. Oxford University Press, New York.

\section{ANEXo 1}

Valores $p$ de la prueba a posteriori Nemenyi para Kruskal-Wallis $(\alpha=0,05)$ de comparaciones múltiples entre módulos en las yemas axilares, separado por sexo predominante de la especie. Valores en negrita indican diferencias significativas $(p<0,05)$. / P-values of the Nemenyi post-hoc test for Kruskal-Wallis $(\alpha=0.05)$ of multiple comparisons between modules in axillary buds, separated by predominant sex of the species. Values in bold indicate significant differences $(p<0.05)$.

\begin{tabular}{lcc}
\hline Sexo predominante & $\begin{array}{c}\text { Comparación } \\
\text { modular }\end{array}$ & Valor $\mathrm{p}$ \\
\hline Femenino & B vs C & $\mathbf{0 , 0 0 2 9}$ \\
Femenino & B vs D & 0,1566 \\
Femenino & C vs D & $<0,0001$ \\
Masculino & B vs C & 0,6347 \\
Masculino & B vs D & 0,0571 \\
Masculino & C vs D & $\mathbf{0 , 0 0 3 7}$ \\
\hline
\end{tabular}

Received: 29.04.2019

Accepted: 20.08 .2020 